\title{
Estudio estructural de interacciones intra- e intermoleculares basadas en puentes de hidrógeno en piridin-2,6-dicarboxamidas
}

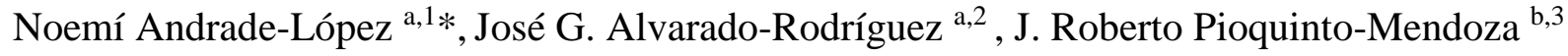

\begin{abstract}
a Área Académica de Química. Universidad Autónoma del Estado de Hidalgo. Ciudad Universitaria, Carretera Pachuca-Tulancingo km 4.5. Colonia Carboneras, Mineral de la Reforma, Hoo. C.P. 42184, México.

${ }^{b}$ Facultad de Química. Universidad Autónoma de Yucatán, Calle 43613 x Calle 90, Colonia Inalámbrica, Mérida, Yuc. C. P. 97069, México
\end{abstract}

\section{Resumen}

En el presente trabajo de investigación se describe el estudio de las interacciones por puente de hidrógeno en piridin-2,6dicarboxamidas de fórmula general $\left\{(2,6-\mathrm{CONRH})_{2}\left(\mathrm{C}_{5} \mathrm{H}_{3} \mathrm{~N}\right)\right\}\left[\mathrm{R}=\mathrm{H}(\mathbf{1}) ; \mathrm{R}=\mathrm{CH}_{3}(\mathbf{2}) ; \mathrm{R}=\mathrm{CH}_{2} \mathrm{CH}_{3}(\mathbf{3}) ; \mathrm{R}=2-\mathrm{Br}-\mathrm{C}_{6} \mathrm{H}_{4}(\mathbf{4})\right.$ y $\mathrm{R}=$ $\mathrm{CH}_{2}\left(2-\mathrm{C}_{5} \mathrm{H}_{4} \mathrm{~N}\right)(\mathbf{5})$ ] caracterizadas mediante Resonancia Magnética Nuclear de ${ }^{1} \mathrm{H}$ y espectroscopía infrarroja. Para las amidas 1-5 se estableció que, tanto en solución como en estado sólido, predominan interacciones intermoleculares del tipo $\mathrm{NH}^{\circ} \mathrm{OH}_{2}, \mathrm{NH}^{\circ} \cdot \mathrm{OC}$ y $\mathrm{NH} \cdots \mathrm{OSMe}_{2}$, así como intramoleculares $\mathrm{NH} \cdots \mathrm{N}$. En las amidas 1-5, los puentes de hidrógeno intramoleculares formados entre el átomo de hidrógeno del grupo $\mathrm{NH}$ amídico y el átomo de nitrógeno piridínico $(\mathrm{NH} \cdots \mathrm{N})$ permiten la formación de anillos de cinco miembros. La presencia de puentes de hidrógeno en estos compuestos contribuye a la forma amida, como el tautómero principal a su estructura.

Palabras Clave: Enlace de hidrógeno, RMN, infrarrojo, difracción de rayos X de monocristal, piridin-2,6-dicarboxamidas.

\section{Introducción}

Las amidas son importantes para el desarrollo de procesos de reconocimiento molecular, para los sistemas anfitriónhuésped y para el diseño supramolecular (Kamlesh \& Weck, 2008), por lo que en las últimas décadas ha aumentado el número de estudios relacionados con la evaluación de sus interacciones intra- e intermoleculares, así como del análisis estructural relacionado con la determinación de su conformación en solución y en estado sólido. Estas caracterizaciones se fundamentan en técnicas espectroscópicas como el infrarrojo (IR) (Gellman et al., 1991), Resonancia Magnética Nuclear (RMN) (Benchabane et al., 2009) y por métodos de difracción de rayos $\mathrm{X}$ de monocristal (Özdem et al., 2012).

La estructura de las amidas primarias y secundarias puede ser representada a través de los contribuyentes tautoméricos amida e iminol, figura 1 , mientras que en amidas terciarias las

\footnotetext{
${ }^{\text {a }}$ Profesora Investigadora de Tiempo Completo

*Autor en correspondencia: nandrade@uaeh.edu.mx

a ${ }^{2}$ Profesor Investigador de Tiempo Completo igar@uaeh.edu.mx

b 33rofesor de Tiempo Completo, jpioquinto@uady.mx
}

estructuras canónicas I y II mostradas en la figura 2 son los contribuyentes principales.<smiles>[R]N=C(O)c1cccc(C(O)=N[R])n1</smiles>

$\mathbf{R}=\mathbf{H}$, alquilo, arilo

Figura 1. Equilibrio tautomérico amida-iminol

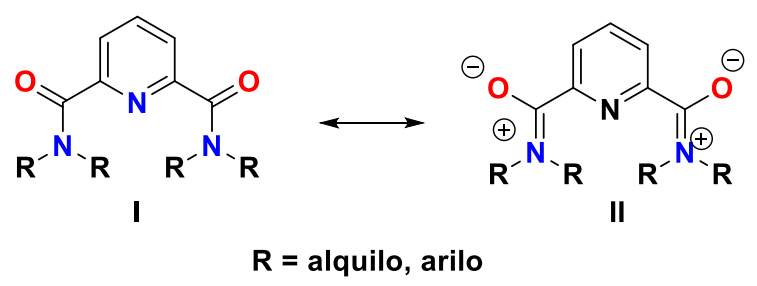

Figura 2. Estructuras resonantes para el grupo amida 
Como se muestra en ambas figuras, los contribuyentes iminol y iónico II producen una reducción en el orden de enlace $\mathrm{C}-\mathrm{O}$, mientras que el orden de enlace $\mathrm{C}-\mathrm{N}$ aumenta. Asimismo, los contribuyentes amida y neutro $\mathbf{I}$ originan un aumento en el orden de enlace $\mathrm{C}-\mathrm{O}$ y la reducción, por ende, del orden de enlace C-N. A estos contribuyentes se les atribuye que los enlaces $\mathrm{C}-\mathrm{O}$ y $\mathrm{C}-\mathrm{N}$ presenten un orden de enlace intermedio de 1.5, permitiendo que las amidas presenten a temperatura ambiente una barrera rotacional baja en torno al enlace C-N a temperatura ambiente (15-21 $\mathrm{kcal} / \mathrm{mol}$ ) (Suezawa et al., 1988). Por lo anterior, la estructura de las amidas puede ser descrita por los rotámeros $E, Z$ cuando los sustituyentes en el átomo de nitrógeno son diferentes, (González et al., 2015) figura 3. Los datos cristalográficos obtenidos para amidas muestran que el átomo de nitrógeno es plano, reforzando la existencia de los equilibrios mostrados en las figuras 1 y 2, (Kaminski et al., 2009) y de la isomería $E, Z$. Para amidas secundarias como las que se encuentran contenidas en péptidos y proteínas el isómero $E$ es el más favorecido energéticamente. Sin embargo, es necesario resaltar que el predominio de isómeros depende, además, de la agregación molecular y de las interacciones por enlaces por puente de hidrógeno que pueden estar presentes en el empaquetamiento cristalino y en solución (Kaminski et al., 2009; Özdem et al., 2012).

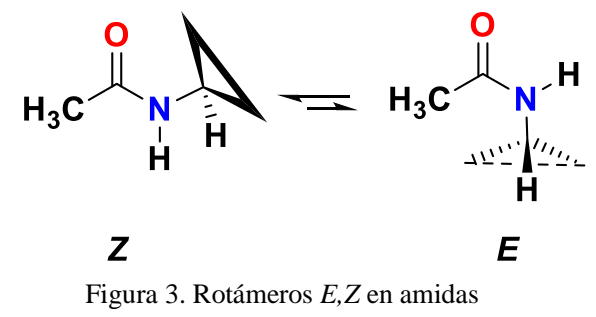

\section{Materiales y Métodos}

\subsection{Materiales y equipo}

Los reactivos piridin-2,6-dicarboxamida (1), metilamina (2 $\mathrm{M}$, en metanol), etilamina ( $2 \mathrm{M}$, metanol), 2 bromoanilina, trietilamina, 2-aminometilpiridina, dibencilamina, dicloruro de piridin-2,6-dicarboxamida, carbonato de potasio, cloroformo, diclorometano y dimetilsulfóxido deuterado (DMSO- $\mathrm{d}_{6}$ ) fueron adquiridos comercialmente (Sigma Aldrich). Todas las manipulaciones se llevaron a cabo utilizando disolventes previamente purificados mediante métodos convencionales y por técnicas Schlenk. Los puntos de fusión fueron determinados en un aparato Mel-Temp II y se reportan sin corregir. Los espectros de IR fueron obtenidos en un espectrofotómetro FT- 200 Perkin Elmer en el intervalo de 4,000 a $400 \mathrm{~cm}^{-1}$, en pastillas de $\mathrm{KBr}$. Los espectros de RMN de ${ }^{1} \mathrm{H}$ fueron obtenidos a $399.78 \mathrm{MHz}$, en un espectrómetro Varian VNMRS 400 utilizando DMSO- $\mathrm{d}_{6}$ como disolvente. Los desplazamientos químicos de ${ }^{1} \mathrm{H}$ se reportan con respecto a la frecuencia interna del tetrametilsilano (TMS). Los experimentos de temperatura variable de ${ }^{1} \mathrm{H}$ se obtuvieron en un intervalo de temperatura de 145 a $25( \pm 0.2){ }^{\circ} \mathrm{C}$ y con una resolución de señal de $\pm 0.02 \mathrm{~Hz}$

\subsection{Método general para la síntesis de las amidas $\mathbf{2}-\mathbf{5}$.}

Para la preparación de los compuestos 2-5 se utilizó una metodología similar a la descrita para la síntesis de amidas análogas (Marlin et al., 2000). A una solución de dicloruro de piridin-2,6-dicarboxamida $\left(\mathrm{C}_{7} \mathrm{H}_{3} \mathrm{NO}_{2} \mathrm{Cl}_{2}\right)$ en $35 \mathrm{~mL}$ de cloroformo $\left(\mathrm{CHCl}_{3}\right)$ se le agregó la amina $N$-sustituida correspondiente; la reacción se dejó en un baño de hielo por un periodo de 30 minutos. Posteriormente se refluyó durante 5 horas. Al término de la reacción se evaporó el disolvente a presión reducida. El sólido obtenido se disolvió en $20 \mathrm{~mL}$ de agua destilada y se neutralizó con $\mathrm{K}_{2} \mathrm{CO}_{3}$. Finalmente fue extraído con 3 porciones de $20 \mathrm{~mL}$ de diclorometano y el disolvente fue eliminado a presión reducida, formándose sólidos de color blanco para 2-4 y un sólido beige para $\mathbf{5}$, esquema 1 .

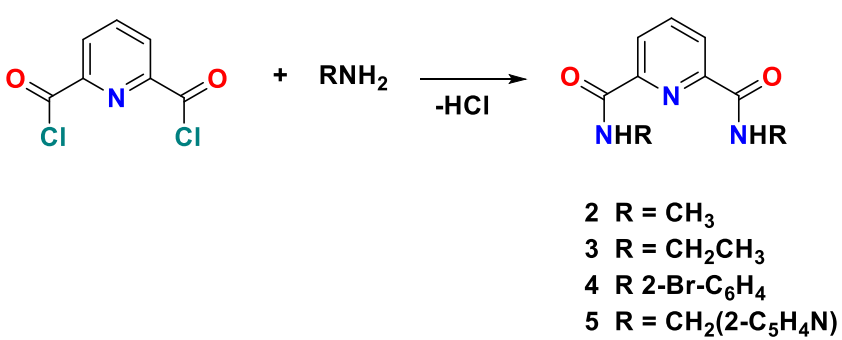

Esquema 1. Síntesis de las amidas $\mathbf{2}-\mathbf{5}$

\subsection{Datos experimentales para las amidas $\mathbf{1 - 5}$}

1. P. f. $317^{\circ} \mathrm{C}$. IR (KBr) cm ${ }^{-1}: v=\left(\mathrm{NH}_{2}\right) 3407,3231$ (f); (CO) 1671,1659 . Datos de RMN de ${ }^{1} \mathrm{H}\left(\delta\right.$ en ppm, DMSO-d $\left.{ }_{6}\right): 8.91$ (s, $\left.2 \mathrm{H}, \mathrm{NH}_{2}\right) .8 .16$ (m, 3H, H2, H3). 7.74 (s, 2H, $\mathrm{NH}_{2}$ ).

2. $2.00 \mathrm{~g}$ (9.80 mmoles) de dicloruro de piridin-2,6dicarboxamida, $14.71 \mathrm{~mL}$ (29.41 mmol) de metilamina $2 \mathrm{M}$ en metanol, sólido blanco. $1.76 \mathrm{~g}(93 \%)$, p. f. $180-182{ }^{\circ} \mathrm{C}$. Análisis elemental calculado para $\left(\mathrm{C}_{9} \mathrm{H}_{11} \mathrm{~N}_{3} \mathrm{O}_{2}\right) \mathrm{C}, 56.0 ; \mathrm{H}, 5.7$; $\mathrm{N}, 2$ 1.8. Encontrado C, 55.9; H, 5.7; N, 19.7. IR ( $\mathrm{KBr}) \mathrm{cm}^{-1}$ : v $=(\mathrm{NH}) 3323,3296 ;(\mathrm{CO}) 1687,1659$. Datos de RMN de ${ }^{1} \mathrm{H}(\delta$ en ppm, DMSO-d 6 ): 9.29 (q, $\left.2 \mathrm{H}, \mathrm{NH},{ }^{3} J=4.85 \mathrm{~Hz}\right) .8 .15(\mathrm{~m}$, $3 \mathrm{H}, \mathrm{H} 3, \mathrm{H} 2) .2 .89\left(\mathrm{~d}, 6 \mathrm{H}, \mathrm{CH}_{3} \mathrm{~N},{ }^{3} \mathrm{~J}=4.85 \mathrm{~Hz}\right)$

3. $2.00 \mathrm{~g}(9.80 \mathrm{mmol})$ de dicloruro de piridin-2,6dicarboxamida, $14.71 \mathrm{~mL}(29.41 \mathrm{mmol})$ de etilamina $2 \mathrm{M}$, sólido blanco. $1.63 \mathrm{~g}(75 \%)$, p. f. $153-156{ }^{\circ} \mathrm{C}$. Análisis elemental calculado para $\left(\mathrm{C}_{11} \mathrm{H}_{15} \mathrm{~N}_{3} \mathrm{O}_{2}\right) \mathrm{C}, 59.7 ; \mathrm{H}, 6.8 ; \mathrm{N}$, 19.0. Encontrado C, 59.6; H, 6.8; N, 18.5. IR (KBr) cm $\mathrm{cm}^{-1}: \mathrm{v}=$ (NH) 3327, 3282; (CO) 1678, 1649. Datos de RMN de ${ }^{1} \mathrm{H}(\delta$ en ppm, DMSO-d $\left.\mathrm{d}_{6}\right)$ : $9.35\left(\mathrm{t}, 2 \mathrm{H}, \mathrm{NH},{ }^{3} J=4.85 \mathrm{~Hz}\right) .8 .15(\mathrm{~m}$, $3 \mathrm{H}, \mathrm{H} 2, \mathrm{H} 3) .3 .42\left(\mathrm{td}, 4 \mathrm{H}, \mathrm{CH}_{2} \mathrm{~N},{ }^{3} J=6.50 ;{ }^{3} \mathrm{~J}=4.85 \mathrm{~Hz}\right) .1 .16$ (t, $6 \mathrm{H}, \mathrm{CH}_{3},{ }^{3} \mathrm{~J}=6.50 \mathrm{~Hz}$ )

4. $1.00 \mathrm{~g}(4.90 \mathrm{mmol})$ de dicloruro de piridin-2,6dicarboxamida, $1.86 \mathrm{~g}$ (10.78 $\mathrm{mmol})$ de 2-bromoanilina, 1.09 g (10.7 mmol) de trietilamina, sólido blanco. $2.01 \mathrm{~g}(83 \%)$, p. f. 193-195 ${ }^{\circ} \mathrm{C}$. Análisis elemental calculado para $\left(\mathrm{C}_{19} \mathrm{H}_{13} \mathrm{Br}_{2} \mathrm{~N}_{3} \mathrm{O}_{2}\right) \mathrm{C}, 48.0 ; \mathrm{H}, 2.8 ; \mathrm{N}, 8.8$. Encontrado C, 47.9; $\mathrm{H}, 2.6 ; \mathrm{N}, 8.9$. IR $(\mathrm{KBr}) \mathrm{cm}^{-1}: v=(\mathrm{NH}) 3362$, 3243; (CO) 1698. Datos de RMN de ${ }^{1} \mathrm{H}$ ( $\delta$ en ppm, DMSO-d $\mathrm{d}_{6}$ ): 10.95 (s, $2 \mathrm{H}, \mathrm{NH}) .8 .35$ (m, 3H, H2, H3). 7,78 (dd, $2 \mathrm{H}, \mathrm{H} 10,{ }^{3} \mathrm{~J}=8.02$, $\left.{ }^{4} J=1.20 \mathrm{~Hz}\right) .7 .67\left(\mathrm{dd}, 2 \mathrm{H}, \mathrm{H} 7,{ }^{3} J=7.84,{ }^{4} J=1.29 \mathrm{~Hz}\right)$. 
5. $2.00 \mathrm{~g}(9.80 \mathrm{mmol})$ de dicloruro de piridin-2,6dicarboxamida, $2.22 \mathrm{~mL} \quad(21.56 \mathrm{mmol})$ de $2-$ aminometilpiridina, sólido beige. $2.76 \mathrm{~g}(81 \%)$, p.f. 121-123 ${ }^{\circ} \mathrm{C}$. Análisis elemental calculado para $\left(\mathrm{C}_{19} \mathrm{H}_{17} \mathrm{~N}_{5} \mathrm{O}_{2} \cdot \mathrm{H}_{2} \mathrm{O}\right) \mathrm{C}$, 62.5; H, 5.2; N, 19.2. Encontrado C, 62.6; H, 5.0; N, 19.2. IR $(\mathrm{KBr}) \mathrm{cm}^{-1}: v=(\mathrm{NH}) 3350,3297$; (CO) 1683, 1664. Datos de RMN de ${ }^{1} \mathrm{H}\left(\delta\right.$ en ppm, DMSO-d 6 ): 10.05 (t, 2H, NH, ${ }^{3} J=6.59$ Hz). 8.51 (ddd, 2H, H9, ${ }^{3} J=4.83 ;{ }^{4} J=1.71 ;{ }^{5} J=0.86 \mathrm{~Hz}$ ). 8.23 (m, 3H, H2, H3). 7.75 (td, $\left.2 \mathrm{H}, \mathrm{H} 7,{ }^{3} \mathrm{~J}=7.60 ;{ }^{4} \mathrm{~J}=1.71 \mathrm{~Hz}\right)$. 7.35 (dd, 2H, H6, $\left.{ }^{3} J=7.60 ;{ }^{4} J=0.80 \mathrm{~Hz}\right) .7 .26(\mathrm{ddd}, 2 \mathrm{H}, \mathrm{H} 8$, $\left.{ }^{3} \mathrm{~J}=7.60 ;{ }^{4} \mathrm{~J}=4.83 ;{ }^{5} \mathrm{~J}=0.80 \mathrm{~Hz}\right) .4 .70\left(\mathrm{~d}, 4 \mathrm{H}, \mathrm{CH}_{2} \mathrm{~N},{ }^{3} \mathrm{~J}=6.59\right.$ $\mathrm{Hz}$ )

\section{Resultados y discusión}

\subsection{Evaluación de puentes de hidrógeno por IR}

La amida primaria $\mathbf{1}$ y las amidas secundarias 2-5 mostraron dos bandas de vibración de tensión del grupo carbonilo (CO). La primera fue observada en el intervalo de 1698 a $1671 \mathrm{~cm}^{-1}$ y fue asignada al grupo CO de amida libre. Para las amidas 1-5, esta $v_{\mathrm{CO}}$ se observó a frecuencia mayores comparada con la asignada para la amida terciaria $\mathbf{6}\left(v_{\mathrm{CO}}=\right.$ $1638 \mathrm{~cm}^{-1}$ ), en donde II tiene una contribución importante, figura 4. Esta diferencia en frecuencias confirma que la forma amida es el contribuyente principal a la estructura de los compuestos 1-5 en estado sólido, figura 4. La observación de una segunda banda asignada al grupo CO entre 1659 y 1649 $\mathrm{cm}^{-1}$, se ha asociado con la formación de interacciones intermoleculares CO...HN (Marlin et al., 2000).

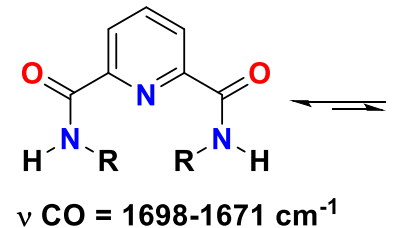

forma amida

forma iminol

1-5 $\mathrm{R}=\mathrm{H}, \mathrm{CH}_{3}, \mathrm{CH}_{2} \mathrm{CH}_{3}, 2-\mathrm{Br}-\mathrm{C}_{6} \mathrm{H}_{5}, \mathrm{CH}_{2}\left(2-\mathrm{C}_{5} \mathrm{H}_{4} \mathrm{~N}\right)$<smiles>[R]N([R])C(=O)c1cccc(C(=O)N([R])CC)n1</smiles><smiles>[R][N+]([R])=C([O-])c1cccc(C([O-])=[N+]([R])[O-])n1</smiles>

I II $\checkmark C O=1638 \mathrm{~cm}^{-1}$

\section{$6 \mathrm{R}=\mathrm{CH}_{2} \mathrm{C}_{6} \mathrm{H}_{5}$}

Figura 4. Contribuyentes principales a la estructura de las amidas 1-6

Los espectros de infrarrojo de las amidas 1-5 mostraron dos bandas para las vibraciones de tensión ( $v$ ) del grupo $\mathrm{NH}$; la primera en el intervalo de 3407 a $3323 \mathrm{~cm}^{-1}$ y la segunda entre 3297 y $3231 \mathrm{~cm}^{-1}$. Las vibraciones de tensión del grupo $\mathrm{NH}$ a frecuencias menores (3297 y $3231 \mathrm{~cm}^{-1}$ ) y su comparación con las del grupo $\mathrm{NH}$ en amidas que presentan interacciones por enlace por puente de hidrógeno (Gellman et al., 1990), sugieren la presencia de este tipo de interacciones en los compuestos 1-5. Estas interacciones pueden ser intermoleculares $\mathrm{y} / \mathrm{o}$ intramoleculares del tipo $\mathrm{O} \cdots \mathrm{HN}$ y
$\mathrm{N} \cdots \mathrm{HN}$ respectivamente, como las descritas en las estructuras cristalinas para 1 y 2 (Marlin et al., 2000), en donde el rotámero $Z, Z$ es el contribuyente principal en estado sólido para 2, figura 5.<smiles>O=C1NC2NC3C(=O)N(C(O)O)C23N1</smiles>

1

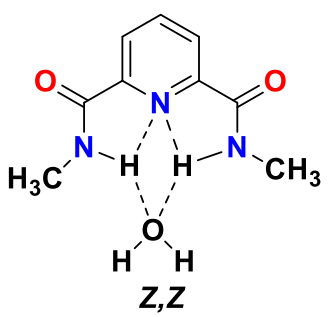

2
Figura 5. Puentes de hidrógeno intra- e intermoleculares reportados en las estructuras cristalinas de $\mathbf{1}$ y $\mathbf{2}$ (Marlin, et al., 2000)

La primera $v_{\mathrm{NH}}$ en $3407 \mathrm{~cm}^{-1}$ para la amida $\mathbf{1}$, se asignó a un NH libre por comparación con valores previamente reportados en el intervalo de 3521 a $3400 \mathrm{~cm}^{-1}$ (Gellman et al., 1990), mientras que la segunda observada hacia frecuencias menores en $3231 \mathrm{~cm}^{-1}$ se atribuyó a un puente de hidrógeno intramolecular $\mathrm{N} \cdots \mathrm{HN}$ (figura 6), los cuales han sido reportados en estado sólido en el intervalo de 3295 a 3230 $\mathrm{cm}^{-1}$ (Alvarez-Hernández et al., 2017); lo anterior es acorde a la interacción intramolecular $\mathrm{N} \cdots \mathrm{HN}$ descrita por difracción de rayos $\mathrm{X}$ de monocristal para esta amida (Marlin et al., 2000).

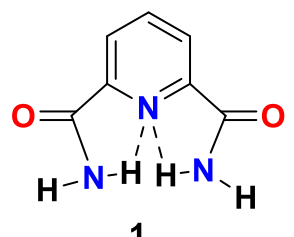

$v$ NH libre $=3407 \mathrm{~cm}^{-1}$

$\checkmark \mathrm{NH}$ puente $=3231 \mathrm{~cm}^{-1}$

Figura 6. Vibraciones de tensión (v) para el grupo NH en el IR en la amida 1

Para las amidas 2-5, la primera banda de vibración de tensión del grupo $\mathrm{NH}$ en el intervalo de 3362 a $3323 \mathrm{~cm}^{-1}$, se atribuyó a asociaciones intermoleculares $\mathrm{CO} \cdots \mathrm{HN}$ por comparación con amidas análogas $\left(v=3373\right.$ a $\left.3292 \mathrm{~cm}^{-1}\right)$ reportadas por Marlin et al. La segunda $v_{\mathrm{NH}}$ se observó en el intervalo de 3297 a $3243 \mathrm{~cm}^{-1}$ y por comparación con las descritas por Alvarez et al., se atribuyeron a puentes de hidrógeno intramoleculares del tipo $\mathrm{N} \cdot \cdot \mathrm{HN}$ como se muestra en la figura 7. Es necesario resaltar, que la determinación por infrarrojo de las $v$ de los grupos $\mathrm{NH}$ asociados a puentes de hidrógeno intermoleculares $\mathrm{CO} \cdots \mathrm{HN}$ e intramoleculares N..HN es factible, debido a que éstos varían entre sí, en un intervalo de 150 a $100 \mathrm{~cm}^{-1}$. Lo anterior se atribuye a que las asociaciones diméricas o poliméricas en los puentes de hidrógeno intermoleculares $\mathrm{CO} \cdots \mathrm{HN}$, como las que se muestran en la figura 7 , repercuten en una mayor constante de fuerza originando que el grupo $\mathrm{NH}$ se desplace hacia frecuencias mayores. 

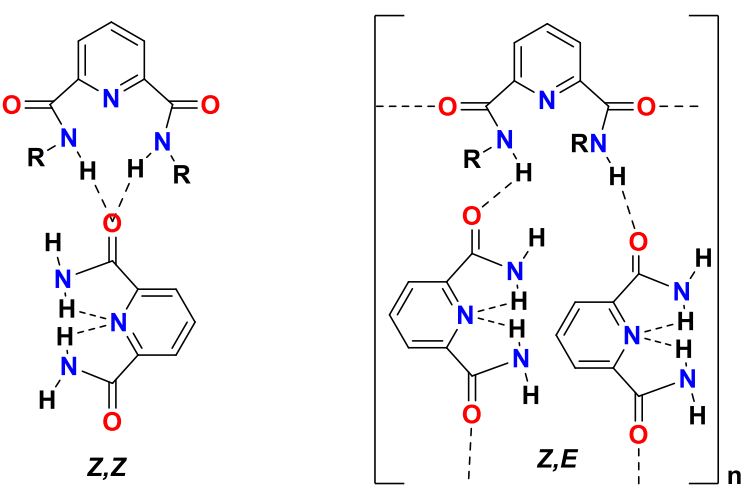

$\checkmark$ NH puente intermolecular $=3362-3323 \mathrm{~cm}^{-1}$ $\checkmark$ NH puente intramolecular $=3297-3243 \mathrm{~cm}^{-1}$

$$
\begin{aligned}
2 \mathrm{R} & =\mathrm{CH}_{3} \\
3 \mathrm{R} & =\mathrm{CH}_{2} \mathrm{CH}_{3} \\
5 \mathrm{R} & =\mathrm{CH}_{2}\left(2-\mathrm{C}_{5} \mathrm{H}_{4} \mathrm{~N}\right)
\end{aligned}
$$

Figura 7. Vibraciones de tensión $(v \mathrm{NH})$ asignadas a puentes de hidrógeno inter- e intramoleculares en las amidas 2-5

\subsection{Evaluación de puentes de hidrógeno por $R M N$ de ${ }^{l} H$}

En los espectros de ${ }^{1} \mathrm{H}$ de los compuestos 1-5 en solución de DMSO- $\mathrm{d}_{6}$, se observó una señal simple en el intervalo de 10.95 a 7.74 ppm para los protones del $\mathrm{NH}$ amídico. La desprotección magnética de los protones de este grupo y por lo tanto su desplazamiento hacia frecuencias mayores se atribuye a la interacción donador-aceptor $\mathrm{NH} \cdots \mathrm{N}$ (Gellman et al., 1991). Para establecer la naturaleza de los puentes de hidrógeno en solución se realizaron experimentos de temperatura variable en el intervalo de 418 a $298 \mathrm{~K}$ por $\mathrm{RMN}$ de ${ }^{1} \mathrm{H}$, utilizando DMSO- $\mathrm{d}_{6}$ como disolvente. Con este método, los valores de desplazamiento químico con respecto a la temperatura $(\Delta \delta / \Delta \mathrm{T})$ mayores a $4 \times 10^{-3} \mathrm{ppm} / \mathrm{K}$ se asocian a la presencia de puentes de hidrógeno intermoleculares, mientras que valores menores o iguales a $3 \times 10^{-3} \mathrm{ppm} / \mathrm{K}$ se asocian a puentes de hidrógeno intramoleculares. Para las amidas 2,3 y 5 se obtuvieron valores de $\Delta \delta / \Delta \mathrm{T}$ de $3.54 \times 10^{-3}$, $4.25 \times 10^{-3}$ y $4.08 \times 10^{-3} \mathrm{ppm} / \mathrm{K}$, respectivamente. Estos valores indican la presencia de puentes de hidrógeno intermoleculares, posiblemente por asociación con el disolvente $\mathrm{NH} \cdots \mathrm{OSMe}_{2}$ (figura 8), en donde los rotámetros $Z, Z$ y $Z, E$ son probables contribuyentes. Para la amida 1 se obtuvieron dos valores de $\Delta \delta / \Delta \mathrm{T}$ debido a que los protones del grupo $\mathrm{NH}_{2}$ tienen ambientes químicos diferentes, por lo que cada protón se observó como una señal doble de dobles en 8.91 y 7.74 ppm. Para el protón en $8.91 \mathrm{ppm}$ se obtuvo un $\Delta \delta / \Delta \mathrm{T}$ de $4.90 \times 10^{-3}$ $\mathrm{ppm} / \mathrm{K}$; por lo que se propone la participación de este grupo $\mathrm{NH}$ en un puente de hidrógeno intermolecular del tipo $\mathrm{NH} \cdots \mathrm{OSMe}_{2}$, similar al propuesto para 2,3 y 5 . Para el protón en $7.74 \mathrm{ppm}$, el valor de $\Delta \delta / \Delta \mathrm{T}$ fue de $3.30 \times 10^{-3} \mathrm{ppm} / \mathrm{K}$; éste último valor se encuentra en un intervalo más cercano al descrito para puentes de hidrógeno intramoleculares con respecto a los obtenidos para las amidas $2, \mathbf{3}$ y $\mathbf{5}$. Con base a esta comparación, a las características estructurales de $\mathbf{1}$ y a las asociaciones intramoleculares $\mathrm{NH} \cdots \mathrm{N}$ previamente reportadas en su estructura cristalina, (Marlin et al., 2000), se propone que este protón participa en un puente de hidrógeno intramolecular del tipo $\mathrm{NH} \cdots \mathrm{N}$, figura 8 .<smiles></smiles><smiles>[R]NC(=O)c1cccc(C(=O)N([R])[CH]O[Si](C)(C)C)n1</smiles><smiles>[R]NC(=O)c1cccc(C(=O)N[C@H]([R1])O[SH](C)C)n1</smiles>

$\mathbf{Z , Z}$ $Z, E$

$$
\begin{aligned}
2 \mathrm{R} & =\mathrm{CH}_{3} \\
3 \mathrm{R} & =\mathrm{CH}_{2} \mathrm{CH}_{3} \\
5 \mathrm{R} & =\mathrm{CH}_{2}\left(2-\mathrm{C}_{5} \mathrm{H}_{4} \mathrm{~N}\right)
\end{aligned}
$$

Figura 8. Puentes de hidrógeno en las amidas $\mathbf{1}$ y 3-5 establecidos en solución de DMSO-d 6

\section{Conclusión}

Los datos espectroscópicos obtenidos en solución y en estado sólido confirman que la forma amida es el contribuyente principal a la estructura de los compuestos 1-5. El predominio de este tautómero se asocia a la presencia de interacciones intra-e intermoleculares por puente de hidrógeno, las cuales favorecen la forma amida en el equilibrio amida-iminol. La naturaleza y el número de asociaciones inter- e intramoleculares establecidas por infrarrojo y por RMN, permiten proponer la contribución de los rotámeros $Z, Z$ y $E, Z$ a la estructura de las amidas 2-5. Considerando que el rotámero $Z, Z$ es el que presenta las menores repulsiones electrónicas en las amidas 2-5 y, a que en 2 es el de mayor contribución en estado cristalino (Marlin et al., 2000), se propone como el rotámero predominante en solución y en estado sólido.

\section{Summary}

\section{STRUCTURAL STUDY OF INTRA- AND INTERMOLECULAR INTERACTIONS BASED ON HYDROGEN BONDING IN PYRIDINE-2,6- DICARBOXAMIDES}

\section{Abstract}

Study of hydrogen bonding in pyridine-2,6-dicarboxamides of general formula $\left\{(2,6-\mathrm{CONRH})_{2}\left(\mathrm{C}_{5} \mathrm{H}_{3} \mathrm{~N}\right)\right\}[\mathrm{R}=\mathrm{H}(\mathbf{1}) ; \mathrm{R}=$ $\mathrm{CH}_{3}(2) ; \mathrm{R}=\mathrm{CH}_{2} \mathrm{CH}_{3}(3) ; \mathrm{R}=2-\mathrm{Br}_{-} \mathrm{C}_{6} \mathrm{H}_{4}(4) ; \mathrm{R}=\mathrm{CH}_{2}(2-$ $\left.\left.\mathrm{C}_{5} \mathrm{H}_{4} \mathrm{~N}\right)(\mathbf{5})\right]$ by NMR and infrared is described. Intermolecular interactions of types $\mathrm{NH} \cdots \mathrm{OH}_{2}, \mathrm{NH} \cdots \mathrm{OC}$ and $\mathrm{NH} \cdot \mathrm{OSMe}_{2}$, and intramolecular $\mathrm{NH} \cdots \mathrm{N}$ interactions for the amides 1-5 in 
solution and solid state were established. In 1-5, the intramolecular hydrogen bonds formed with the hydrogen atoms of the $\mathrm{NH}$ amidic group and the pyridinic nitrogen $(\mathrm{NH} \cdots \mathrm{N})$ yielded the formation of five membered rings. The hydrogen bonds in these compounds enhance the amide form as the main tautomer associated to their structure.

Keywords: Hydrogen bond, ${ }^{1} \mathrm{H}-\mathrm{NMR}$, infrared, X-ray diffraction, pyridine-2,6-dicarboxamides

\section{Agradecimientos}

Este trabajo fue parcialmente financiado por el proyecto "Integración de Redes Temáticas de Colaboración Académica 2015 SEP".

\section{Referencias}

Alvarez-Hernández, J.-A., Andrade-López, N., AlvaradoRodríguez, J. G., Vásquez-Pérez, J. M., Cruz-Borbolla, J., \& Vojtech, J. (2017). Synthesis and structural characterization of 10 Group metal complexes with anionic tridentate $S, N, N$ donor Schiff bases derived from pyridylbenzothiazolines. Polyhedron, 169-179.

Benchabane, Y., Boyer, G., Humbel, S., Alkorta, I., \& Elguero, J. (2009). A theoretical and NMR experimental study of N1,N3-di(3-aminoacridin-6-yl)-isophthalamide and N2,N6-di(3-aminoacridin-6-yl)-2,6-dicarboxamide. $J$. Molec. Struct., 132-137.

Gellman, S. H., Adams, B. R., \& Dado, G. P. (1990). Temperature-Dependent Changes in the Folding Pattern of a Simple Triamide. J. Am. Chem. Soc., 460-461.
Gellman, S. H., Dado, G. P., Liang, C.-B., \& Adam, R. (1991). Conformation-Directing Effects of a Single Intramolecular Amide-Amide Hydrogen Bond: Variable-Temperature NMR and IR Studies on a Homologous Diamide Series. $J$. Am. Chem. Soc., 1164-1173.

González-de-Castro, A., Broughton, H., Martínez-Pérez, J. A., \& Espinosa, J. F. (2015). Conformational Features of Secondary N-Cyclopropyl Amides. J. Org. Chem., 3914-3920.

Kaminski, R., Schilf, W., Cmoch, P., Dziembowska, D., \& Wozniak, K. (2009). On structural and spectroscopic differences between quinoline-2-carboxamides and their Noxides in the solution and solid state. J. Phys. Org. Chem., 857-871.

Kamlesh, P. N., \& Weck, M. (2008). NonCovalent Side Chain Modification. En V. Rotello, \& S. Thayumanavan, Molecular recognition and polymers: Control of Polymer Structure and Self-Assembly. United States of America: Wiley.

Marlin, D. S., Olmstead, M. M., \& Mascharak, P. K. (2000). Extended structures controlled by intramolecular and intermolecular hydrogen bonding: a case study with pyridine-2,6-dicarboxamide, 1,3-benzenedicarboxamide and N,N'-dimethyl-2,6-pyridinedicarboxamide. J. Molec. Struct. , 211-223.

Özdem, N., Dayan, O., Etinkaya, B. C., \& Akgül, C. (2012). Concomitant polymorphism of a pyridine-2,6dicarboxamide derivative in a single space group: Experimental and molecular modeling study. Spectrochim. Acta, Part A, 614-624.

Suezawa, H., Tsuchiya, K.-Y., Tahara, E., Hota, M., \& Suezawa, ,. (1988). Multinuclear NMR and the Rotational Barriers about the C-N Bonds of Several, N,NDisubstituted Arenecarboxamides. Bull. Chem. Soc. Jpn., 4057-4065. 